\title{
Mental Performance in Old Age Dependent on Cortisol and Genetic Variance in the Mineralocorticoid and Glucocorticoid Receptors
}

\author{
Maris Kuningas*, ${ }^{*, 2}$, Roel H de Rijk ${ }^{3,4}$, Rudi GJ Westendorp', Jelle Jolles ${ }^{5}$, P Eline Slagboom ${ }^{6}$ and \\ Diana van Heemst' \\ 'Department of Gerontology and Geriatrics, Leiden University Medical Center, Leiden, The Netherlands; ${ }^{2}$ Department of Biotechnology, Institute \\ of Molecular and Cell Biology, Tartu University, Tartu, Estonia; ${ }^{3}$ Department of Psychiatry, Leiden University Medical Center, Leiden, The \\ Netherlands; ${ }^{4}$ Division of Medical Pharmacology, Leiden/Amsterdam Center for Drug Research, Leiden University Medical Center, Leiden, The \\ Netherlands; ${ }^{5}$ Department of Psychiatry and Neuropsychology, University Hospital Maastricht, Maastricht, The Netherlands; ${ }^{6}$ Department of \\ Medical Statistics, Section of Molecular Epidemiology, Leiden University Medical Center, Leiden, The Netherlands
}

\begin{abstract}
Depression and cognitive decline have been associated with changes in circulating cortisol concentrations. Cortisol exerts its functions through mineralocorticoid (MR) and glucocorticoid (GR) receptors. However, data on the influence of variations in the MR and GR genes on depressive symptoms and cognitive functioning in older adults are scarce. Therefore, we explored the impact of MR-2 I5G/C, MR-II80V, GR-ER22/23EK, GR-N363S, and GR-Bcll polymorphisms on these end points in the population-based Leiden 85-plus Study. This prospective study includes 563 participants aged 85 years and older, with a mean follow-up of 4.2 years. In this study, high morning cortisol levels (per I SD cortisol) associated with impairments in global cognitive functioning $(p=0.002)$ at baseline (age 85). These impairments were mainly attributable to lower attention $(p=0.057)$ and slower processing speed $(p=0.014)$. Similar effects were also observed during follow-up (age 85-90), where participants with higher cortisol levels (per I SD cortisol) had impaired global cognitive functioning $(p=0.003)$, as well as impairments in attention $(p=0.034)$ and processing speed $(p=0.013)$. Changes in depressive symptoms were observed for the MR-II80V single-nucleotide polymorphism (SNP), where during follow-up the prevalence of depressive symptoms was higher in the I80V-allele carriers $(p=0.049)$ compared to noncarriers. Dependent on these polymorphisms, no differences in overall and in specific domains of cognitive functioning were observed. In conclusion, the MR-II 80V SNP has a specific effect on depressive symptoms, independent from cognitive functioning, and other polymorphisms in the MR and GR genes. In contrast, these genetic variants in the MR and GR genes do not influence cognitive functioning in old age.

Neuropsychopharmacology (2007) 32, I295- I30 I. doi: I 0. I 038/sj.npp. I 30 I260; published online 29 November 2006
\end{abstract}

Keywords: cognition; depressive symptoms; mineralocorticoid receptor; glucocorticoid receptor; cortisol; polymorphisms

\section{INTRODUCTION}

In response to a real or imagined threat the organism reacts by eliciting a stress response in order to cope with the stressor. In humans, cortisol is the primary active stress hormone that mediates counter-responses to stress, aimed to re-establish homeostasis and coordinate behavioral adaptations (de Kloet et al, 1998, 2005; Munck et al, 1984). Cortisol exerts its effects through mineralocorticoid (MR) (NR3C2) and glucocorticoid (GR) (NR3C1) receptors,

\footnotetext{
* Correspondence: M Kuningas, Department of Gerontology and Geriatrics C2-R, Leiden University Medical Center (LUMC), PO Box 9600, Albinusdreef 2, 2300 RC Leiden, The Netherlands, Tel: + 31 (0) 7I 5266640, Fax: + 31 (0) 715248159 ,

E-mail: M.Kuningas@lumc.nl

Received 22 May 2006; revised and ; accepted 6 October 2006
}

which are abundantly co-expressed in the neurons of limbic structures (Herman, 2003). MRs, which have 10 times higher affinity for cortisol than GRs, determine the threshold or sensitivity of the stress system and thus the onset of the stress response (de Kloet et al, 2005). GRs, on the other hand, represent the slower mode that promotes the termination of the stress response (de Kloet et al, 2005). Balancing these two systems is essential for cell homeostasis, mental performance, and health.

Various studies have shown that stress-responsiveness is highly variable among human subjects, and an inadequate stress response increases vulnerability for disease. Changes in the stress hormone system have been shown to play a role in the development of depression (Belanoff et al, 2001b; Holsboer, 2000, 2001). In addition, changes in the circulating cortisol concentrations have been associated with impairments in various cognitive domains, including 
attention, perception, and memory (Jameison and Dinan, 2001; Lupien et al, 2005; Newcomer et al, 1999). In terms of memory, excess cortisol levels have been shown to impair mainly declarative and not nondeclarative memory (Lupien et al, 2005).

As MRs and GRs mediate the cortisol signal, variations in these genes may introduce changes in cortisol signaling dynamics, and thereby lead to changes in mood and cognitive function. In the MR gene, the $215 \mathrm{G} / \mathrm{C}$ and I180V single-nucleotide polymorphisms (SNPs) have been shown to change cortisol signaling in vitro (Arai et al, 2003), whereas in the GR gene the ER22/23EK, N363S, and BclI SNPs have been shown to change HPA-axis reactivity after a dexamethasone suppression test or a psychosocial stressor (Huizenga et al, 1998; van Rossum et al, 2002, 2003). In addition, the ER22/23EK variant has been associated with major depression in two studies (van Rossum et al, 2006; van West et al, 2006). To date, there are no publications that examine the role of these MR and GR variants in cognitive functioning and as yet no study has assessed the effects of variants in the MR gene on mood. Furthermore, a combined effect of the genetic variants in the MR and GR genes may have a different effect on depressive symptoms and cognitive functioning, owing to the interplay between these genes.

In this study, we examined the influence of cortisol levels and variants in the MR and GR genes on overall cognitive functioning, attention, processing speed, immediate and delayed memory, and on depressive symptoms in old age. All analyses were performed in the prospective populationbased Leiden 85-plus Study. Since in old age cognitive decline and depressive symptoms are more prevalent, the effects of a lifelong exposure to changes in cortisol signaling owing to polymorphisms should be more easily detectable on these end points.

\section{SUBJECTS AND METHODS}

\section{Subjects}

The Leiden 85-plus Study is a population-based prospective study of inhabitants of the city Leiden, The Netherlands. All 85-year-old inhabitants were invited to participate. There were no selection criteria related to health or demographic characteristics. The study population consists of 599 participants, all Caucasians and members of the 19121914 birth cohorts, who were enrolled in the month of their 85th birthday between 1997 and 1999 (Bootsma-van der Wiel et al, 2002). For the present study DNA was available for 563 participants. The Medical Ethical Committee of the Leiden University Medical Center approved the study and informed consent was obtained from all participants or their guardian.

\section{Cognitive Function and Depressive Symptoms}

Global cognitive functioning was assessed in all participants with the Mini-Mental State Examination (MMSE) (Folstein et al, 1975). Participants with a MMSE score 19 points or higher were subjected to additional tests to measure attention (Stroop Test) (Klein et al, 1997), processing speed (Letter Digit Coding Test, LDT) (Houx et al, 2002), immediate recall memory (Word Learning Test Immediate Recall, WLTI), delayed recall memory (Word Learning Test Delayed Recall, WLTD) (Brand and Jolles, 1985) and depressive symptoms (15-item Geriatric Depression Scale, GDS-15) (De Craen et al, 2003). The tests assessing specific domains of cognitive functioning were not administered in subjects with a MMSE score of 18 points or lower, because diminished reliability and validity in subjects with severe cognitive impairment. All participants were visited annually for re-measurement of cognitive functioning during a mean follow-up period of 4.2 years. Parallel versions of the tests were used. Details of cognitive testing are described elsewhere (Houx et al, 2002).

\section{Cortisol Measurement at Baseline}

All blood samples were drawn in the morning before $11 \mathrm{AM}$. The median time between venipuncture and centrifugation in our laboratory was $50 \mathrm{~min}$ (interquartile range: 30$70 \mathrm{~min}$ ). Nonfasting plasma cortisol levels were determined by a fluorescence polarization immunoassay using the Abbott TDx (Abbott Laboratories, Abbott Park, IL) according to the manufacturer's instructions. The within-assay coefficient of variation was below $5 \%$ at different levels.

\section{Possible Confounders}

Socio-demographic characteristics, such as sex and level of education were considered as possible confounders. Education was divided into two levels: a lower education level, including individuals without schooling or with only primary school education (less than 6 years of schooling), and those with a higher education level (6 years or more of schooling). Health related correlates were assessed at baseline (age 85), and included drug use, obtained from the pharmacist's registers, and chronic diseases. Data on the use of oral corticosteroids were available for all participants. Chronic diseases included cardiovascular disease, diabetes mellitus, chronic obstructive pulmonary disease, arthritis, malignancy, dementia, and Parkinson's disease. Data were obtained at baseline from a structured questionnaire and based on the diagnoses of the general practitioner.

\section{Genotyping}

The GR-ER22/23EK polymorphism consists of two SNPs in codons 22 (rs6189) and 23 (rs6190). The MR-I180V (rs5522), GR-ER22/23EK, GR-N363S (rs6195), and GR-BclI (van Rossum et al, 2003) variants were genotyped using an Assay-by-Design (Applied Biosystems, Foster City, CA), consisting of PCR primers and TaqMan MGB probes, on an ABI 7900 HT with real-time PCR (Applied Biosystems). MR215G/C (rs2070951) was genotyped using the MassArray platform according to the protocols of the manufacturer (Sequenom, San Diego, CA).

\section{Statistical Analysis}

Allele and genotype frequencies were calculated and analyzed for deviation from Hardy-Weinberg equilibrium using the $\chi^{2}$ test. Cortisol levels were converted into $z$-scores ((individual level-mean level)/SD) in order to normalize 
the data and provide comparable estimates per 1SD increase in cortisol level. The cross-sectional (age 85) and longitudinal (age 85-90) associations between baseline cortisol levels, cognitive functioning, and depressive symptoms were analyzed using a linear regression and a linear mixed model, respectively. Differences in cortisol levels dependent on polymorphisms were assessed with a univariate general linear model. Associations between genotypes, cognitive functioning, and depressive symptoms were analyzed using the linear mixed model, estimating the overall mean difference in cognitive functioning, or depressive symptoms during follow-up. In multivariate analyses, the other polymorphisms were added to the model as independent covariates. The analyses of cognitive functioning were adjusted for sex and level of education, and the analyses of depressive symptoms were adjusted for sex and presence of chronic diseases. All these analyses were also performed with additional adjustment for cortisol levels or chronic diseases. The analyses were performed with SPSS statistical software, version 12.0 (Chicago, IL).

\section{RESULTS}

Baseline characteristics of the participants are shown in Table 1. From the 563 participants, 12 were excluded from the analyses owing to the use of corticosteroids. Global cognitive functioning was assessed in all participants using MMSE. Some $460(83 \%)$ scored 19 points or more on MMSE, and these subjects performed additional tests, measuring attention, processing speed, immediate and delayed recall memory, and depressive symptoms. All 551 subjects were genotyped for the MR-215G/C, MR-I180V, GR-ER22/23EK, GR-N363S, and GR-BclI polymorphisms. The genotype distributions and resulting allelic frequencies of the variants were in agreement with the distribution predicted by the Hardy-Weinberg equilibrium, and in case of the GR gene similar to the frequencies observed in other studies. This is the first study reporting allele frequencies for the SNPs in the MR gene. Because of the low allele frequencies of the SNPs (Table 1), the heterozygous and homozygous minor allele carriers of all the polymorphisms were combined. However, for the MR-215G/C and GR-BclI variants, the analyses were also repeated using all genotypes, as these polymorphisms have higher allele frequencies.

Global cognitive functioning, attention, processing speed, memory, and depressive symptoms were assessed at baseline and re-examined annually during a mean follow-up period of 4.2 years. During follow-up, there was a significant increase in depressive symptoms and a decline in cognitive functioning in all participants (all $p<0.001)$. The influence of plasma cortisol levels on depressive symptoms and cognitive functioning was assessed at baseline (age 85) and also during follow-up (age 85-90). The cross-sectional analysis at age 85 revealed that higher cortisol levels (per $1 \mathrm{SD}$ cortisol) were associated with impairments in overall cognitive performance. This was observed in the whole study population $(p=0.008)$, as well as in the restricted sample (MMSE $\geqslant 19$ points) $(p=0.002)$ (Table 2$)$. These differences were attributable to lower attention $(p=0.057)$ and slower processing speed $(p=0.014)$ (Table 2$)$. Similar
Table I Baseline Characteristics of Study Participants

\begin{tabular}{|c|c|}
\hline Number & 551 \\
\hline $\mathrm{Age}^{\mathrm{a}}$ & $85(-)$ \\
\hline Female (\%) & $365(66 \%)$ \\
\hline Low level of education (\%) & $356(65 \%)$ \\
\hline Chronic diseases & $39|/ 46|$ \\
\hline Cortisol $(\mu \mathrm{mol} / /)^{\mathrm{a}}$ & $0.49(0.15)$ \\
\hline MMSE (points) ${ }^{\mathrm{a}}$ & $23.9(6.27)$ \\
\hline MMSE $\geqslant 19$ points (\%) & $460(83 \%)$ \\
\hline
\end{tabular}

$\begin{array}{ll}\text { Specific domains of cognition }^{\mathrm{a}} & \\ \text { Stroop Test (seconds) } & 82.4(34.3) \\ \text { LDT (digits) } & 17.1(7.12) \\ \text { WLTI (pictures) } & 23.9(6.00) \\ \text { WLTD (pictures) } & 8.65(2.76) \\ \text { GDS-I5 (points) }^{a} & 2.32(2.44)\end{array}$

Polymorphisms (minor allele frequency)

MR-2I5G/C

0.47

$M R-1180 \mathrm{~V}$

0.14

GR-ER22/23EK

0.03

GR-N363S

0.05

GR-BCll C/G

0.34

GDS-15, Geriatric Depression Scale 15-item; LDT, Letter Digit Coding Test; MMSE, Mini-Mental State Examination; WLTI, Word Learning Test Immediate Recall; WLTD, Word Learning Test Delayed Recall.

a Data presented as means with SD.

effects were also observed during follow-up (age 85-90), where participants with higher cortisol levels (per 1SD cortisol) had impaired global cognitive functioning $(p=0.004)$, attention $(p=0.034)$ and processing speed $(p=0.013)$ (Table 2$)$. The overall difference in cognitive functioning and depressive symptoms during follow-up dependent on cortisol levels (two groups based on median cortisol level $0.47 \mu \mathrm{mol} / \mathrm{l}$ ) is shown in Figure 1.

The influence of MR and GR gene polymorphisms on plasma cortisol levels is shown in Table 3. Plasma cortisol levels were associated with the $215 \mathrm{G} / \mathrm{C}$ variant in the MR gene, where the C-allele carriers had lower cortisol levels than noncarriers $(p=0.008)$ (Table 3$)$. In addition, for the GR-BclI C/G SNP, a trend for an increase in cortisol levels in $\mathrm{G}$-allele carriers was observed $(p=0.084)$ (Table 3$)$.

Cognitive functioning and the prevalence of depressive symptoms were also analyzed dependent on the MR and GR gene polymorphisms. Differences in depressive symptoms were observed for the MR-I180V SNP, where carriers of the $180 \mathrm{~V}$-allele had more depressive symptoms than noncarriers $(p=0.049)$ (Table 4, Figure 2). This difference increased after adjusting for cortisol levels ( 0.54 points, SE $(0.27)$, and $p=0.043)$. On the other hand, the analyses with cognitive functioning dependent on the polymorphisms revealed no differences, either in overall (Table 4) or in specific domains of cognitive functioning (data not shown). The results remained unaltered after the analyses with a multivariate model, or adjustment for depressive symptoms and cortisol concentrations (data not shown). The analyses 
Table 2 Cortisol, Cognitive Functioning and Depressive Symptoms at Baseline (Age 85), and during the Annual Follow-Up (Age 85-90)

Age 85

Age 85-90

Difference per I SD cortisol

Difference per I SD cortisol

\begin{tabular}{|c|c|c|c|c|}
\hline & & \\
\hline & Estimate (SE) & $p$-value & Estimate (SE) & p-value \\
\hline \multicolumn{5}{|l|}{ All participants $(n=551)$} \\
\hline MMSE (points) & $-0.67(0.25)$ & 0.008 & $-0.78(0.27)$ & 0.004 \\
\hline \multicolumn{5}{|l|}{ Restricted sample $(n=460)^{a}$} \\
\hline MMSE (points) & $-0.40(0.13)$ & 0.002 & $-0.37(0.12)$ & 0.003 \\
\hline LDT (digits) & $-0.79(0.32)$ & 0.014 & $-0.74(0.30)$ & 0.013 \\
\hline WLTI (pictures) & $-0.39(0.29)$ & 0.187 & $-0.38(0.28)$ & 0.186 \\
\hline WLTD (pictures) & $-0.21(0.14)$ & 0.129 & $-0.19(0.13)$ & 0.160 \\
\hline GDS-I5 (points) & $0.18(0.12)$ & 0.146 & $0.10(0.13)$ & 0.424 \\
\hline
\end{tabular}

GDS- 15, Geriatric Depression Scale I5-item; LDT, Letter Digit Coding Test; MMSE, Mini-Mental State Examination; WLTI, Word Learning Test Immediate Recall; WLTD, Word Learning Test Delayed Recall.

${ }^{a}$ Restricted to participants who scored on MMSE $\geqslant 19$ points.

p-values in blod $<0.05$.
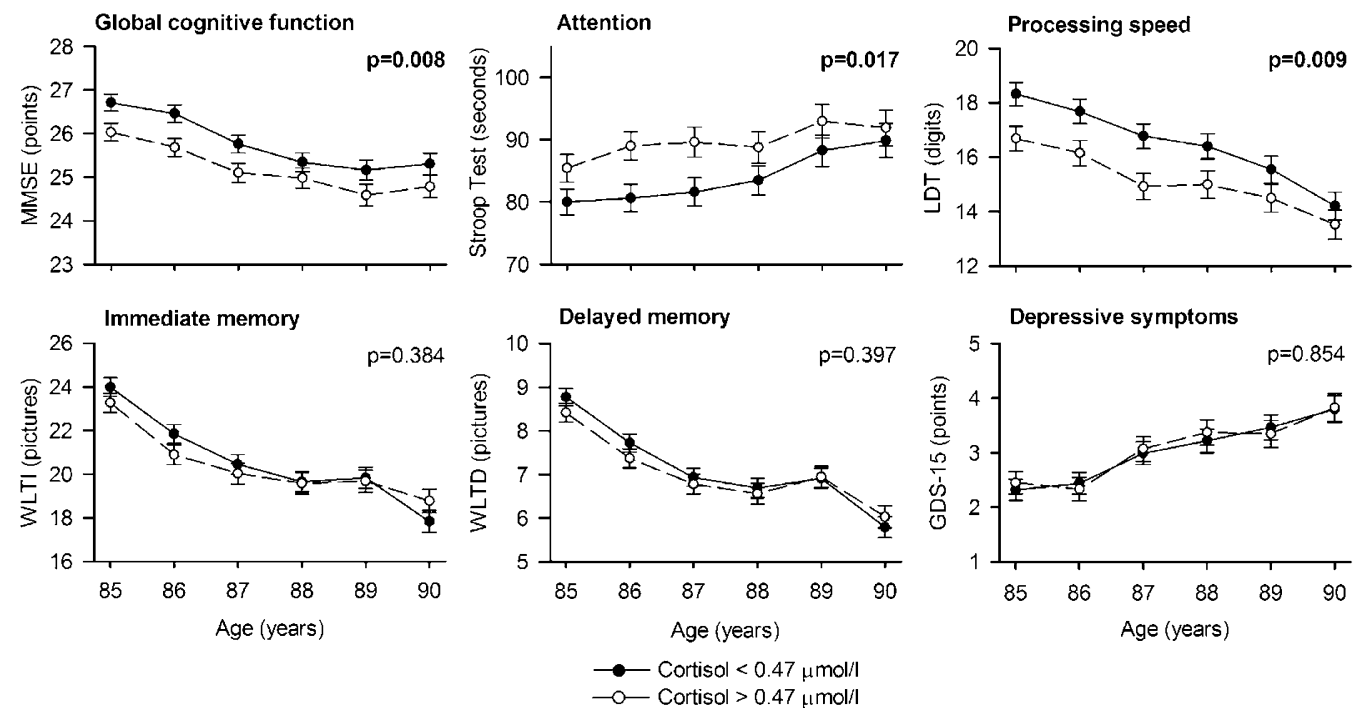

Figure I Cognitive functioning and depressive symptoms during the mean 4.2-year follow-up period in participants with low ( $<0.47 \mu$ mol/l) and high $(>0.47 \mu \mathrm{mol} / \mathrm{l})$ cortisol levels. The $p$-values represent the overall mean differences in cognitive functioning and mood during the follow-up between the two groups.

using all the MR-215G/C and GR-BclI genotypes did not provide any extra information.

All the cognitive functioning analyses were repeated with additional adjustment for chronic diseases, as participants with higher cortisol levels had a higher prevalence of chronic diseases at baseline. This adjustment only marginally changed the results (data not shown).

\section{DISCUSSION}

In this study, we assessed the impact of cortisol levels and of variations in the MR and GR genes on cognitive function and depressive symptoms. The results revealed associations between high-cortisol levels and cognitive impairment, but not with depressive symptoms. In contrast, the prevalence of depressive symptoms was dependent on a variation in the MR gene, where carriers of the $180 \mathrm{~V}$-allele had more depressive symptoms compared to the noncarriers. This association remained unchanged after taking into account the influence of the other polymorphisms in the MR and GR genes. Variations either in the MR gene or in the GR gene were not associated with cognitive function.

Similar to observations in other studies, we found an association between high-cortisol levels and cognitive impairments. These differences were observed at baseline (age 85) and also during follow-up period (age 85-90). Impairments in global cognitive functioning, owing to high 
Table 3 Cortisol Levels $(\mu \mathrm{mol} / \mathrm{l})$ Dependent on the MR and GR Polymorphisms

\begin{tabular}{|c|c|c|c|c|c|}
\hline & \multicolumn{2}{|c|}{ Noncarriers } & \multicolumn{2}{|c|}{ Carriers } & \multirow[b]{2}{*}{$p$-value } \\
\hline & $n$ & Mean (SE) & $n$ & Mean (SE) & \\
\hline MR-2I5G/C & 135 & $0.52(0.01)$ & 376 & $0.48(0.01)$ & 0.008 \\
\hline MR-II80V & 394 & $0.49(0.01)$ & 145 & $0.48(0.01)$ & 0.423 \\
\hline GR-ER22/23EK & 504 & $0.49(0.01)$ & 33 & $0.49(0.03)$ & 0.895 \\
\hline GR-N363S & 494 & $0.49(0.01)$ & 50 & $0.50(0.02)$ & 0.491 \\
\hline GR-Bcll C/G & 234 & $0.47(0.01)$ & 288 & $0.50(0.01)$ & 0.084 \\
\hline
\end{tabular}

p-values in blod $<0.05$.

Table 4 Cognitive Functioning and Depressive Symptoms during the Mean 4.2 year Follow-Up Period Dependent on the MR and GR Polymorphisms

\begin{tabular}{llll}
\hline & $\begin{array}{c}\text { Noncarriers } \\
\text { Mean (SE) }\end{array}$ & $\begin{array}{c}\text { Carriers } \\
\text { Mean (SE) }\end{array}$ & p-value \\
\hline MMSE (points) & & & \\
MR-2I5G/C & $25.2(0.25)$ & $25.6(0.15)$ & 0.179 \\
MR-II80V & $25.5(0.15)$ & $25.5(0.23)$ & 0.926 \\
GR-ER22/23EK & $25.5(0.14)$ & $25.8(0.48)$ & 0.458 \\
GR-N363S & $25.5(0.13)$ & $25.3(0.43)$ & 0.776 \\
GR-BCll C/G & $25.5(0.19)$ & $25.4(0.17)$ & 0.809 \\
& & & \\
GDS-I5 (points) & & & \\
MR-2I5G/C & $3.12(0.24)$ & $2.98(0.15)$ & 0.594 \\
MR-II80V & $2.90(0.15)$ & $3.42(0.23)$ & $\mathbf{0 . 0 4 9}$ \\
GR-ER22/23EK & $3.02(0.13)$ & $3.53(0.48)$ & 0.297 \\
GR-N363S & $3.04(0.13)$ & $2.55(0.4 I)$ & 0.248 \\
GR-BCII C/G & $3.26(0.19)$ & $2.89(0.17)$ & 0.133 \\
\hline
\end{tabular}

GDS-I5, Geriatric Depression Scale 15-item; MMSE, Mini-Mental State

Examination.

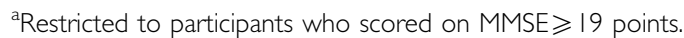

$p$-values in blod $<0.05$.

cortisol, were observed in the whole study population, and also in the restricted sample (MMSE $\geqslant 19$ points). In the restricted sample, the estimates were without exception smaller, however they had increased significance. This implies that by excluding severely cognitively impaired participants we lose some power but increase specificity, as these participants may have impaired cognition caused by other factors than high cortisol. From the specific domains of cognitive functioning, attention, and speed of information processing were most affected, whereas for memory and mood only a modest influence was observed. Intriguingly, in literature high-cortisol levels have been mainly associated with memory impairments and depression (Holsboer, 2000, 2001; Lupien et al, 2005). Most of these studies have been conducted with exogenous administration of glucocorticoids or with depressed patients, with higher cortisol concentrations than observed in this study. Therefore, it might be that cortisol levels higher than the average values in older adults are necessary to affect memory

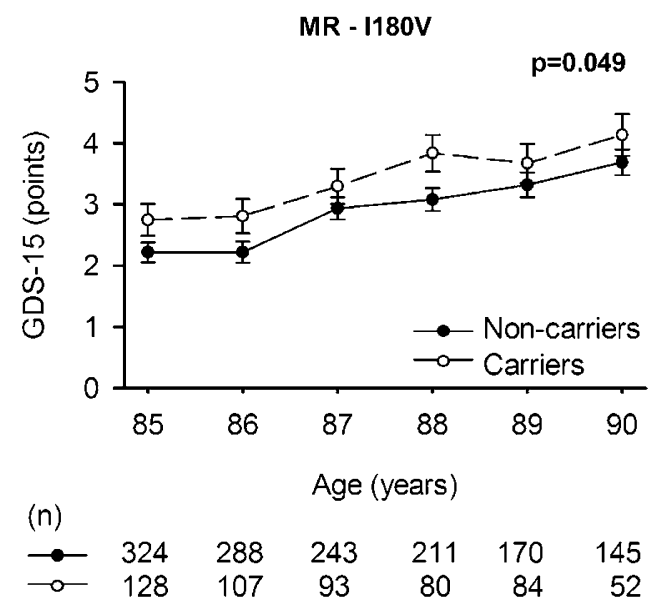

Figure 2 Prevalence of depressive symptoms in noncarriers and carriers of the MR-II 80V polymorphism during the mean 4.2-year follow-up period. The $p$-value represents the overall mean difference in the GDS- 15 scores during follow-up between the two groups. (n) Represents the number of participants.

function and depressive symptoms. However, the lack of associations could also be related to the single cortisol measurement, or to the tests used in this study. It has been shown, that not the acute basal cortisol levels, but rather the change in cortisol levels over time predicts cognitive impairments in aging (Belanoff et al, 2001a). In addition, it has been shown that in some cases, cortisol effects on cognitive performance are very difficult to detect with the use of standardized, less sensitive cognitive tests. Thus, it is plausible that the memory tests used in this study are not sensitive enough to detect cognitive impairments related to cortisol.

MRs have been implicated in the control of HPA-axis activity, as they determine the threshold of stress system's activation (de Kloet et al, 2005). To date, there is no information on how polymorphisms in the MR gene influence these functions. In this study, we found that plasma cortisol levels were lower in C-allele carries of the $215 \mathrm{G} / \mathrm{C}$ SNP in the MR gene. As the $215 \mathrm{G} / \mathrm{C}$ SNP precedes the start codon of the MR gene (Arai et al, 2003), it could interfere with the translational start site and thus, lead to a less efficient translation and change in MR-A and MR-B balance (Pascual-Le Tallec et al, 2004). However, the influence of the different MR isoforms on cortisol levels is not known. From the other MR polymorphisms, we found that the I180V amino-acid change in the MR gene was associated with higher prevalence of depressive symptoms. It has been reasoned that this SNP changes MR's transactivational properties (Arai et al, 2003), which might change stress responsiveness and lead to increased vulnerability to develop depressive symptoms. On the other hand, carriers of the MR-I180V polymorphism did not display any deficits in overall or in specific domains of cognitive functioning, which suggests that the polymorphism specifically influences depressive symptoms and not cognitive functioning. The mechanisms behind cortisol-mediated effects on cognitive functioning and depressive symptoms are most probably different, and modulated by different neuronal pathways and/or contextual conditions (Lupien et al, 2005). 
Previously, it has been shown that the GR gene polymorphisms alter HPA-axis responsiveness and thereby cortisol sensitivity. The ER22/23EK variation has been associated with resistance to cortisol (van Rossum et al, 2002 ), whereas the N363S and BclI polymorphisms were shown to increase the sensitivity to cortisol (Huizenga et al, 1998; van Rossum et al, 2002, 2003). We reasoned, that the increased resistance to cortisol attained by the ER22/23EK variant would protect against the damaging effects of highcortisol levels on cognitive decline and depression, whereas increased cortisol sensitivity would have opposite effects. In this study, we found no influences of the analyzed polymorphisms on plasma cortisol levels, or on cognitive functioning. For depressive symptoms, only a trend for an influence by the ER22/23EK variant was observed. In contrast, the 22/23EK-allele carriers tended to have more depressive symptoms than noncarriers, without any deficits in cognitive functioning. The lack of a significant difference in depressive symptoms between the ER22/23EK carriers and noncarriers in our study may result from the low number of participants carrying the variant allele. Despite that, the trend observed in our study, is in accordance with two recent studies, showing an enrichment of the 22/23EKallele in patients with major depression (van Rossum et al, 2006; van West et al, 2006). It could be that in our study the lack of a distinct influence of the GR gene SNPs on cognitive functioning and mood is a result of the nonstressful testing conditions. GRs are activated in response to stress and they promote the termination of the stress response and normalization of homeostasis (de Kloet et al, 2005). This suggests that the effects of the polymorphisms are only seen in response to stress or challenge, and that testing in basal conditions does not reveal any differences in cognitive functioning and depressive symptoms.

The response to stress is modulated by the interplay between MRs and GRs, and a combined effect of genetic variants in these genes may have a different effect on depressive symptoms and cognitive functioning. In this study, we attempted to take into account the influence of genetic variability in both the MR and GR genes. However, the multivariate analysis did not add any extra information. The cortisol signaling cascade is complex and involves numerous chaperones, accessory proteins, co-regulators and interacting transcription factors that permit differentiation between the MR- and GR-mediated actions (De Bosscher et al, 2003; Pascual-Le Tallec and Lombes, 2005). For instance, recently it was found that increase in recurrence of depressive episodes is associated with a variant in a gene (FKBP5) that regulates GR activity (Binder et al, 2004). Therefore, not only genes directly involved in stress-induced signaling pathways, but genes regulating their activity also appear to be involved in the development of these disorders, and their actions should be analyzed in more detail.

Strengths of the present study include the populationbased sample of the oldest old with high incidence of depression and cognitive decline, and the annual repeated assessment of depressive symptoms and various cognitive domains. A major limitation of the study is the one time cortisol measurement. Cortisol is secreted diurnally, and the single measurement of cortisol over an entire day of the circadian cortisol secretion, considerably weakens the value of this measure. Cortisol levels measured only once could be confounded by acute effects, and not reflect the basal concentration. It has been shown, that cortisol is relatively stable from day to day but it is not indisputably stable across the years. Therefore, the results on the predictability of baseline cortisol levels on cognitive decline over the years should be interpreted with care. Another limitation of the study is the lack of data on HPA-axis reactivity, or on other parameters that reflect the stress system's activity.

In conclusion, this is the first study looking into the influence of polymorphisms in the MR and GR genes on mood and cognitive functioning in older adults. We found an association between high baseline cortisol levels and impairments in cognitive functioning during follow-up. However, no differences in cognitive functioning were observed dependent on the polymorphisms in the MR and GR genes. On the other hand, the I $180 \mathrm{~V}$ variant in the MR gene influenced the prevalence of depressive symptoms, independently from cognitive functioning.

\section{ACKNOWLEDGEMENTS}

This work was supported by an IOP grant (Innovative Orientated Research) from the Dutch Ministry of Economic Affairs (Grant number IGE010114), and by the Centre for Medical Systems Biology (CMSB), which is a center of excellence approved by the Netherlands Genomics Initiative/Netherlands Organization for Scientific Research (NWO). The study was also supported by a Marie Curie Fellowship of the European Community program EUROGENDIS 'The Genetic Basis of Disease' for MK, under contract number QLGA-GH-00-60005-59. All authors have seen and agreed with the contents of the manuscript and none of the authors have any financial interests to disclose.

\section{REFERENCES}

Arai K, Nakagomi Y, Iketani M, Shimura Y, Amemiya S, Ohyama K et al (2003). Functional polymorphisms in the mineralocorticoid receptor and amirolide-sensitive sodium channel genes in a patient with sporadic pseudohypoaldosteronism. Hum Genet 112: 91-97.

Belanoff JK, Gross K, Yager A, Schatzberg AF (2001a). Corticosteroids and cognition. J Psychiatr Res 35: 127-145.

Belanoff JK, Kalehzan M, Sund B, Fleming Ficek SK, Schatzberg AF (2001b). Cortisol activity and cognitive changes in psychotic major depression. Am J Psychiatry 158: 1612-1616.

Binder EB, Salyakina D, Lichtner P, Wochnik GM, Ising M, Putz B et al (2004). Polymorphisms in FKBP5 are associated with increased recurrence of depressive episodes and rapid response to antidepressant treatment. Nat Genet 36: 1319-1325.

Bootsma-van der Wiel A, van Exel E, De Craen AJ, Gussekloo J, Lagaay AM, Knook DL et al (2002). A high response is not essential to prevent selection bias: results from the Leiden 85plus study. J Clin Epidemiol 55: 1119-1125.

Brand N, Jolles J (1985). Learning and retrieval rate of words presented auditorily and visually. J Gen Psychol 112: 201-210.

De Bosscher K, Vanden Berghe W, Haegeman G (2003). The interplay between the glucocorticoid receptor and nuclear factor-[kappa]B or activator protein-1: molecular mechanisms for gene repression. Endocr Rev 24: 488-522.

De Craen AJ, Heeren TJ, Gussekloo J (2003). Accuracy of the 15item geriatric depression scale (GDS-15) in a community sample of the oldest old. Int J Geriatr Psychiatry 18: 63-66. 
de Kloet ER, Joels M, Holsboer F (2005). Stress and the brain: from adaptation to disease. Nat Rev Neurosci 6: 463-475.

de Kloet ER, Vreugdenhil E, Oitzl MS, Joels M (1998). Brain corticosteroid receptor balance in health and disease. Endocr Rev 19: 269-301.

Folstein MF, Folstein SE, McHugh PR (1975). 'Mini-mental state'. A practical method for grading the cognitive state of patients for the clinician. J Psychiatr Res 12: 189-198.

Herman JP (2003). Central mechanisms of stress integration: hierarchical circuitry controlling hypothalamo-pituitaryadrenocortical responsiveness. Front Neuroendocrinol 24: 151-180.

Holsboer F (2000). The corticosteroid receptor hypothesis of depression. Neuropsychopharmacology 23: 477-501.

Holsboer F (2001). Stress, hypercortisolism and corticosteroid receptors in depression: implications for therapy. J Affect Disord 62: 77-91.

Houx PJ, Shepherd J, Blauw GJ, Murphy MB, Ford I, Bollen EL et al (2002). Testing cognitive function in elderly populations: the PROSPER study. PROspective Study of Pravastatin in the Elderly at Risk. J Neurol Neurosurg Psychiatry 73: 385-389.

Huizenga NA, Koper JW, de Lange P, Pols HA, Stolk RP, Burger H et al (1998). A polymorphism in the glucocorticoid receptor gene may be associated with and increased sensitivity to glucocorticoids in vivo. J Clin Endocrinol Metab 83: 144-151.

Jameison K, Dinan TG (2001). Glucocorticoids and cognitive function: from physiology to pathophysiology. Hum Psychopharmacol 16: 293-302.

Klein M, Ponds RW, Houx PJ, Jolles J (1997). Effect of test duration on age-related differences in Stroop interference. J Clin Exp Neuropsychol 19: 77-82.

Lupien SJ, Maheu FS, Weekes N (2005). Glucocorticoids: effects on human cogntion. In: Steckler T, Kalin NH, Reul JMHM (eds).
Handbook of Stress and the Brain. Elsevier Science: Amsterdam. pp 387-402.

Munck A, Guyre PM, Holbrook NJ (1984). Physiological functions of glucocorticoids in stress and their relation to pharmacological actions. Endocr Rev 5: 25-44.

Newcomer JW, Selke G, Melson AK, Hershey T, Craft S, Richards K et al (1999). Decreased memory performance in healthy humans induced by stress-level cortisol treatment. Arch Gen Psychiatry 56: 527-533.

Pascual-Le Tallec L, Demange C, Lombes M (2004). Human mineralocorticoid receptor A and B protein forms produced by alternative translation sites display different transcriptional activities. Eur J Endocrinol 150: 585-590.

Pascual-Le Tallec L, Lombes M (2005). The mineralocorticoid receptor: a journey exploring its diversity and specificity of action. Mol Endocrinol 19: 2211-2221.

van Rossum EF, Binder EB, Majer M, Koper JW, Ising M, Modell S et al (2006). Polymorphisms of the glucocorticoid receptor gene and major depression. Biol Psychiatry 59: 681-688.

van Rossum EF, Koper JW, Huizenga NA, Uitterlinden AG, Janssen JA, Brinkmann AO et al (2002). A polymorphism in the glucocorticoid receptor gene, which decreases sensitivity to glucocorticoids in vivo, is associated with low insulin and cholesterol levels. Diabetes 51: 3128-3134.

van Rossum EF, Koper JW, Van Den Beld AW, Uitterlinden AG, Arp P, Ester W et al (2003). Identification of the BclI polymorphism in the glucocorticoid receptor gene: association with sensitivity to glucocorticoids in vivo and body mass index. Clin Endocrinol (Oxford) 59: 585-592.

van West D, Van Den EF, Del Favero J, Souery D, Norrback KF, Van Duijn C et al (2006). Glucocorticoid receptor gene-based SNP analysis in patients with recurrent major depression. Neuropsychopharmacology 31: 620-627. 